tik, Geostrategie und Geoökonomie die analytische Schärfe fehlt - sie werden implizit so ausgeweitet, dass sie als Analyseraster der transatlantischen Beziehungen nicht mehr fungieren können. Gleiches gilt für das Schlagwort des Pragmatismus, das wohl die Bedenken des Autors spiegelt, ein passendes Etikett für die seit dem Herbst 2008 von der Finanz- und Wirtschaftskrise dominierten transatlantischen Beziehungen zu finden.

Und so predigt Fröhlich das Offensichtliche - dies aber mit detaillierter Sachkenntnis. Allen, denen die Entwicklung des transatlantischen Verhältnisses der letzten Jahre unter den Nägeln brennt, ist dieses Buch zu empfehlen. Sie werden mit den Einschätzungen eines Kenners der transatlantischen Beziehungen konfrontiert, der sich der Entfremdungstendenzen der beiden Kontinente ohne die Klammer der konkreten Bedrohung im Grunde bewusst ist und der sich eine Revitalisierung der Beziehungen wünscht. Die nach Auffassung des Rezensenten geopolitische Realität - nämlich die sicherheitspolitische, ökonomische und kulturelle Orientierung der Vereinigten Staaten nach Mittel- und Südamerika, vor allem aber nach Asien - spielt jedoch die Beschwörung gemeinsamer Verletzlichkeit als Nukleus einer atlantischen Sonderbeziehung in die Kulisse.

Manfred Groß

\section{Shibuya, Eric Y. Demobilizing Irregular}

Forces. Cambridge/Malden. John Wiley \& Sons 2012. 167 Seiten. 17,99€.

Auf der Agenda externer Akteure in fragilen oder kollabierten Staaten beziehungsweise in Post-Konfliktlagen figuriert die Demobilisierung irregulärer bewaffneter Gruppen an vorderster Stelle. Die Umsetzung von Friedensabkommen, die (Wieder-)Herstellung eines staatlichen Gewaltmonopols und die Neutralisierung, Transformation beziehungsweise Integration von irregulären Gewaltakteuren gelten als zentrale Motive für Programme der Entwaffnung, Demobilisierung und Reintegration (DD\&R). Die knappe Überblicksdarstellung von Shibuya, Associate Professor am „US Marine Corps Command and Staff College", behandelt die Geschichte und Evolution von DD\&R-Programmen, deren Einzelbestandteile und die damit verbundenen „Herausforderungen“.

Während DD\&R-Programme lange als Generalschlüssel für die Transformation von Gewaltkonflikten durch UNMissionen überschätzt wurden, beginnt Shibuya mit einer nüchternen Feststellung: Belastbare Evidenz für die Friedensbildung durch DD\&R gebe es nicht (10); bestenfalls könne man kostbare Zeit kaufen. DD\&R-Programme gingen davon aus, dass irreguläre Kräfte illegitim seien; was aber, wenn diese Sicherheit und Dienstleistungen offerierten, weil der Staat gänzlich versage? Shibuya verweist damit auf den Kontext schwacher oder kollabierter Staatlichkeit, in denen DD\&R-Programme häufig stattfinden, und auf die negative Bewertung von Aufständischen, die gegen die Regierung operieren, im Unterschied zu Milizen, die auf Seiten der Regierung (oder internationaler Akteure) kämpfen und deshalb als legitim betrachtet werden, obschon sie das staatliche Gewaltmonopol ebenfalls unterminieren.

Der Autor macht auf eine Fülle inhärenter Widersprüche und Zielkonflikte aufmerksam, darunter die problematische Zuerkennung des Kombattanten- 
status beziehungsweise die Anerkennung von Milizen, den häufig exklusiven Fokus auf die Einsammlung von Kleinwaffen, die sozialen und ökonomischen Bedürfnisse von Kombattanten, deren Bevorzugung gegenüber anderen bedürftigen Gruppen und die mögliche Entstehung eines sekundären Waffenmarktes. Vor allem aber hebt er die Unsicherheit für Kombattanten und Gemeinschaften hervor, die infolge von DD\&R-Programmen ihre bisherige Machtbasis verlieren. Nichtstaatliche Gewaltakteure können zur organisierten Kriminalität übergehen oder ihre militärische in politische Macht transformieren. Der Erfolg von DD\&R-Programmen hinge vom Sicherheitsgefühl der Kombattanten und den Gemeinschaften ab, er könne nicht getrennt von der Sicherheitssektorreform betrachtet werden. Darüber hinaus dürfe der Erfolg von DD\&R-Maßnahmen nicht mechanisch an der Zahl eingesammelter Waffen gemessen werden (47). Generell stellt Shibuya deshalb die Abfolge - Entwaffnung vor Demobilisierung - infrage. Ein Kult der Waffen beziehungsweise „militarisierte Maskulinität“ (55) mag den sozialen Status von Waffenbesitzern definieren, weshalb der Autor wiederholt den nötigen Wandel der Gewaltkulturen und die Kriegsmüdigkeit als Voraussetzung für DD\&R-Programme hervorhebt. Er beschreibt die Voraussetzungen und Formen der sozialen, wirtschaftlichen, psychologischen und politischen (Re-)Integration von Kombattanten in traumatisierten und zutiefst gespaltenen Gesellschaften und stellt Verknüpfungen der DD\&R-Agenda zur Übergangsjustiz beziehungsweise der „Versöhnung“ her. Ziel müsse es sein, die unmittelbar nach Kriegsende verständliche Vorzugsbehandlung von (Ex-)Kombattanten zu- gunsten von Gemeinschafts- bzw. Entwicklungsvorhaben $\mathrm{zu}$ überwinden (126f.).

Der „Erfolg“ von DD\&R-Programmen, so Shibuya, sei letztlich eine Frage der Wahrnehmung der Legitimität von (Ex-)Kombattanten beziehungsweise der Akzeptanz von deren Agenda durch die Bevölkerung. Werden die Anliegen nicht-staatlicher Gewaltakteure als legitim angesehen und politisch befriedigt oder aufgrund organisierter Kriminalität weithin abgelehnt? Damit definiert Shibuya den Erfolg von DD\&R-Programmen letztlich über die Umsetzung der Agenda von (Ex-)Kombattanten beziehungsweise von deren Unterstützern und nicht primär über die Auflösung beziehungsweise Transformation nichtstaatlicher Gewaltakteure an sich. Sein Fazit mündet in einem allgemeinen Plädoyer für die Berücksichtigung der sozialen und politischen Gewaltkontexte und für Flexibilität in der Gestaltung von DD\&RProgrammen.

Der Autor listet sachkundig die Vielfalt der Tücken und Fallstricke auf, aber es bleibt über weite Strecken beim Problemaufriss. Das Buch wird als „erste umfassende Einführung in DD\&R“ " angepriesen - eine Übertreibung, die zum Teil auch einem veralteten Literaturstand des Werkes geschuldet ist. Shibuya mischt luzide Einsichten aus DD\&R-Programmen mit Sollensansprüchen, es handelt sich jedoch nicht um eine systematische, komparative politikwissenschaftliche Aufarbeitung. Das Buch ist durchweg flüssig geschrieben und reich bestückt mit illustrativen Fallbeispielen aus Interventionskontexten der letzten 15 Jahre sowie Verweisen auf weiterführende Literatur. Erfrischend sind vor allem das wache Problembewusstsein des Autors und die 
Entmystifizierung des normativ überladenen Sujets. Das bündige Sachbuch dürfte vornehmlich als Einführung für Studenten, für Vorbereitungskurse (etwa am Zentrum für internationale Friedenseinsätze), für Fachkräfte der Entwicklungszusammenarbeit und die politische Bildungsarbeit hilfreich sein.

Andreas Heinemann-Grüder

\section{Comaroff, Jean und John L. Comaroff.}

Der Süden als Vorreiter der Globalisierung. Neue postkoloniale Perspektiven. Frankfurt a.M.. Campus 2012. 278 Seiten. $29,90 €$.

Wie heutzutage zunehmend üblich, handelt es sich bei dem vorliegenden Band um eine gerahmte Ansammlung früher veröffentlichter Aufsätze. Das Buch ist gerade für Politikwissenschaftler_innen höchst relevant, allerdings nicht wegen seiner Kernthese, sondern wegen der kenntnisreichen Darstellung südafrikanischer politischer Systeme und Gesellschaftsbilder. Der Kernthese vorgelagert sind einige andere Thesen, die allerdings in postkolonial halbwegs gebildeten Kreisen kaum für einen erhöhten Pulsschlag sorgen: Die Autor_innen behaupten, dass „die Herausbildung der Moderne ein welthistorischer Prozess war" (16) (und kein europäischer Alleingang), dass „die heutigen weltgeschichtlichen Prozesse die überkommene Geographie von Peripherie und Zentrum sprengen, indem sie einige der innovativsten und dynamischsten Formen der Wertschöpfung nach Süden [...] verlagern " (ebd.) und dass „die Moderne im Süden nicht als bloßer Ableger oder Doppelgänger des euroamerikanischen, Original' zu begreifen ist" (17). So weit, so zutreffend. Die Autor_innen begnügen sich allerdings nicht mit der Absage an das modernisierungstheoretische Narrativ, sie gehen einen entscheidenden Schritt weiter: Es sei „der Süden [...], in dem die radikal neuen Assemblagen von $\mathrm{Ka}$ pital und Arbeit Gestalt annehmen, der also die Zukunft des Nordens präfiguriert [...] Afrika, Südasien und Lateinamerika scheinen in vieler Hinsicht der euromodernen Welt ein Stück voraus zu sein, als Vorreiter ihrer entstehenden Geschichte“ (23f). Dies allerdings ist offensichtlich nichts anderes als die schlichte Umkehr jenes Narrativs.

Im einzelnen bezieht sich diese Kernthese - die im englischen Untertitel How Euro-America is Evolving Toward Africa deutlicher wird als in der deutschen Übersetzung - auf Auswirkungen einer neoliberalen Globalisierung, die im Süden bereits deutlich sichtbarer, im Norden aber erst im Entstehen begriffen seien. Gemeint sind unter anderem eine neoliberale Transformation von Staatlichkeit (38), eine dementsprechend stark begrenzte Version von Demokratie (48), eine Verrechtlichung von Politik beziehungsweise eine Hinwendung zum Recht als dem Ort politischen Streits (50), vor allem die Verdrängung von Politik auf der Grundlage unterschiedlicher Ideologien durch Politik auf der Grundlage politisierter Identitäten („ID-ologie“, 98) und nicht zuletzt die Herausbildung neuer Formen des politischen Handelns (59). Die in den einzelnen Kapiteln angeführten Belege sind durchaus interessant, aber bei weitem nicht systematisch und umfassend genug, um die These der afrozentrierten Teleologie überzeugend wirken lassen zu können. Weit interessanter sind jedoch die Erkenntnisse der hier versammelten anthropologischen Studien jenseits der Kernthese. Die Ausführungen der Au- 